\title{
Exercise induced stress in horses: Selection of the most stable reference genes for quantitative RT-PCR normalization Katia Cappelli* $^{* 1}$, Michela Felicetti ${ }^{\dagger 1}$, Stefano Capomaccio ${ }^{\dagger 2}$, Giacomo Spinsanti ${ }^{3}$, Maurizio Silvestrelli ${ }^{1}$ and Andrea Verini Supplizi ${ }^{1}$
}

\begin{abstract}
Address: ${ }^{1}$ Department of Pathology, Diagnostic and Veterinary Clinic, University of Perugia, Via San Costanzo 4, 06126 Perugia, Italy, 2Department of Applied Biology, University of Perugia, Borgo XX giugno 74, 06100 Perugia, Italy and ${ }^{3}$ Department of Evolutionary Biology, University of Siena, Via A. Moro 2, 53100 Siena, Italy

Email: Katia Cappelli* - katia.cappelli@unipg.it; Michela Felicetti - michelafelicetti@yahoo.it; Stefano Capomaccio - capemaster@gmail.com; Giacomo Spinsanti - spinsanti@unisi.it; Maurizio Silvestrelli - maurizio.silvestrelli@unipg.it; Andrea Verini Supplizi - andrea.verini@unipg.it

* Corresponding author †Equal contributors
\end{abstract}

Published: 19 May 2008

BMC Molecular Biology 2008, 9:49 doi:10.1 186/1471-2199-9-49

This article is available from: http://www.biomedcentral.com/147I-2199/9/49

(c) 2008 Cappelli et al; licensee BioMed Central Ltd.

This is an Open Access article distributed under the terms of the Creative Commons Attribution License (http://creativecommons.org/licenses/by/2.0), which permits unrestricted use, distribution, and reproduction in any medium, provided the original work is properly cited.
Received: 22 November 2007

Accepted: 19 May 2008

\begin{abstract}
Background: Adequate stress response is a critical factor during athlete horses' training and is central to our capacity to obtain better performances while safeguarding animal welfare.

In order to investigate the molecular mechanisms underlying this process, several studies have been conducted that take advantage of microarray and quantitative real-time PCR (qRT-PCR) technologies to analyse the expression of candidate genes involved in the cellular stress response.

Appropriate application of qRT-PCR, however, requires the use of reference genes whose level of expression is not affected by the test, by general physiological conditions or by inter-individual variability.
\end{abstract}

Results: The expression of nine potential reference genes was evaluated in lymphocytes of ten endurance horses during strenuous exercise. These genes were tested by qRT-PCR and ranked according to the stability of their expression using three different methods (implemented in geNorm, NormFinder and BestKeeper). Succinate dehydrogenase complex subunit A (SDHA) and hypoxanthine phosphoribosyltransferase (HPRT) always ranked as the two most stably expressed genes. On the other hand, glyceraldehyde-3-phosphate dehydrogenase (GAPDH), transferrin receptor (TFRC) and ribosomal protein L32 (RPL32) were constantly classified as the less reliable controls.

Conclusion: This study underlines the importance of a careful selection of reference genes for qRT-PCR studies of exercise induced stress in horses. Our results, based on different algorithms and analytical procedures, clearly indicate SDHA and HPRT as the most stable reference genes of our pool. 


\section{Background}

Knowledge of the molecular mechanisms underlying the stress response in athlete horse is a fundamental prerequisite for planning an appropriate training schedule to obtain better performances, preserve animal welfare and avoid overtraining-syndrome $[1,2]$.

It is universally accepted that moderate physical activity may have beneficial effects in terms of general health conditions and could favour the functioning of the immune system. Conversely, strenuous exercise, like exhaustive endurance races, may have detrimental effects on the immune system, determine changes in the cellular composition of peripheral blood and induce the expression of genes that appear to be related to the overtraining-syndrome [3-5]. The list of candidate genes is nevertheless far from being complete, as the athlete's reaction to exercise is a coordinated response of multiple organ systems, and likely involves multiple and complex regulatory changes: induction of heat shock proteins, inflammatory response modulation (pro and anti-inflammatory cytokines) and generation of reactive oxygen and nitrogen species (ROS and RSN) that, besides their damaging potential, play a crucial role in cellular signalling $[6,7]$.

Since exercise has been shown to be an important factor in regulating immune cells and their functions, and considering that stress evokes inflammatory reactions, lymphocytes are considered the best candidate cell type to study physiological changes associated with exhaustive exercise [5].

Quantitative real-time PCR (qRT-PCR) is the technique of choice when trying to detect modifications in transcription levels in a reliable and reproducible manner. Nevertheless, there are some technical issues that must be taken into account, such as quality and quantification of the starting material, enzyme efficiency, and primer design. Different approaches have been proposed to normalize measurements of expression levels [8], but this is generally done using an internal control gene, known as a ref- erence gene or as housekeeping gene (HKG), under the assumption that this has a constant level of expression in the chosen tissue, is not affected by the treatment and has no inter-individual variability. In addition, the reference gene and the target gene should have similar ranges of expression to avoid analytical problems.

Widely expressed genes like ACTB, GAPDH or R18S are generally preferred, without preliminary analysis of their expression profiles under the specific study conditions $[5,6,9]$. Nevertheless, a number of studies report how commonly accepted HKGs do not always constitute reliable controls [10-14], because of unexpected variation in their expression profiles.

More appropriately, multiple HKGs should be evaluated before their employment, and their stability should be measured in the context of the relevant experimental conditions.

A number of statistical methods have been proposed to evaluate stability of gene expression and select the best HKGs in a given experimental setting $[9,15-18]$.

The aim of this paper is to identify the best reference genes for qRT-PCR experiments investigating horse lymphocyte gene expression in exercise induced stress. Statistical algorithms implemented in geNorm [9], BestKeeper [19], and NormFinder [20] were used.

\section{Results}

To assess which are the most stable genes during strenuous exercise, nine potential HKGs were tested in ten endurance horses with a time course sampling strategy. A qRT-PCR assay, based on SYBR $^{\circledast}$ Green detection, was designed for the transcription profiling of the nine genes (ACTB, B2M, GAPDH, HPRT, R18S, RPL32 SDHA, TFRC and $U B B$, Table 1). The specificity of the amplifications was confirmed by melting curve analyses (Additional files $1,2,3,4,5,6,7,8,9)$. For each assay, a standard curve was generated by using 4 -fold serial dilutions of pooled

Table I: Details of the nine genes evaluated.

\begin{tabular}{|c|c|c|c|}
\hline Gene Symbol & Gene Name & Function & Accession Number \\
\hline ACTB & $\beta$-actin & Cytoskeletal structural protein & $\underline{\text { AF035774 }}$ \\
\hline$B 2 M$ & $\beta$-2-microglobulin & Cytoskeletal protein involved in cell locomotion & $\underline{X 69083}$ \\
\hline GAPDH & glyceraldehyde-3-phosphate dehydrogenase & Glycolytic enzyme & $\underline{\mathrm{AFI}} 57 \mid 26$ \\
\hline HPRT & hypoxanthine phosphoribosyltransferase & Metabolic salvage of purines in mammals & AY372182 \\
\hline$R / 8 S$ & ribosomal RNA I8S & Member of ribosome RNA & A] 311673 \\
\hline RPL32 & ribosomal protein $\mathrm{L} 32$ & Member of ribosomal proteins & CX594263 \\
\hline SDHA & succinate dehydrogenase complex subunit $A$ & $\begin{array}{c}\text { Electron transporter in the TCA cycle and respiratory } \\
\text { chain }\end{array}$ & DQ402987 \\
\hline TFRC & transferrin receptor & Iron uptake & DQ284764 \\
\hline$U B B$ & ubiquitin $\mathrm{B}$ & Protein degradation & $\overline{\mathrm{AF} 506969}$ \\
\hline
\end{tabular}


Table 2: Assay conditions for each of the nine genes evaluated.

\begin{tabular}{|c|c|c|c|c|}
\hline Gene symbol & Primers 5'-3' (forward, reverse) & Amplicon length, bp & PCR efficiency, \% & $\begin{array}{l}\text { Correlation with dilution series } \\
\qquad\left(\mathbf{R}^{2}\right)\end{array}$ \\
\hline ACTB & $\begin{array}{l}\text { GGACCTGACGGACTACCTC } \\
\text { CACGCACGATTTCССТСТC }\end{array}$ & 83 & 95.2 & 0.999 \\
\hline$B 2 M$ & $\begin{array}{l}\text { CCTGCTCGGGCTACTCTC } \\
\text { CATTCTCTGCTGGGTGACG }\end{array}$ & 89 & 100.2 & 1.000 \\
\hline GAPDH & $\begin{array}{l}\text { ATCTGACCTGCCGCCTGGAG } \\
\text { CGATGCCTGCTTCACCACCTTC }\end{array}$ & 68 & 102.6 & 1.000 \\
\hline HPRT & $\begin{array}{l}\text { AATTATGGACAGGACTGAACGG } \\
\text { ATAATCCAGCAGGTCAGCAAAG }\end{array}$ & 121 & 93.9 & 1.000 \\
\hline$R / 8 S$ & $\begin{array}{l}\text { GTCTGCCCTATCAACTTTCG } \\
\text { TTCCTTGGATGTGGTAGCC }\end{array}$ & 119 & 94.1 & 0.998 \\
\hline RPL32 & $\begin{array}{l}\text { GGGAGCAATAAGAAAACGAAGC } \\
\text { CTTGGAGGAGACATTGTGAGC }\end{array}$ & 138 & 97.0 & 1.000 \\
\hline SDHA & $\begin{array}{l}\text { GAGGAATGGTCTGGAATACTG } \\
\text { GCCTCTGCTCCATAAATCG }\end{array}$ & 91 & 96.0 & 0.999 \\
\hline TFRC & $\begin{array}{l}\text { TGGCTACTTGGGCTATTGTAAACG } \\
\text { GGTGGTTCTGTTCCCTCTATCTCC }\end{array}$ & 90 & 97.6 & 0.998 \\
\hline$U B B$ & $\begin{array}{l}\text { TTCGTGAAGACCCTGACC } \\
\text { CCTTATCCTGGATCTTGGC }\end{array}$ & 91 & 99.4 & 0.999 \\
\hline
\end{tabular}

cDNAs. As shown in Table 2, linear correlation coefficients $\left(\mathrm{R}^{2}\right)$ varied from 0.998 to 1.000 and PCR efficiencies (E) ranged between 93.9 and 102.6\%.

\section{Expression levels of candidate reference genes}

Cycle threshold values (Cts) for the nine HKGs tested ranged between 17.9 (ACTB) and 26.6 (TFRC). The gene encoding $18 \mathrm{~S}$ rRNA is largely over expressed (Ct 9.1) compared to the protein coding genes. Each single control gene appeared to be equally expressed in the tested cDNA samples, and the variations of the Ct values (calculated for each single gene in the ten horse individuals subtracting the Min Ct from the Max Ct values) was always smaller than one (Figure 1).

\section{Data analysis}

Profiles obtained for each horse and HKG were analysed using three different methods, implemented in the software geNorm, NormFinder and BestKeeper.

GeNorm provides a ranking of the tested genes, based on their expression stability, determining the two most stable HKGs for normalization purposes. Selected HKGs were ranked according to the stability measure $M$ (average pairwise variation of each gene against all others), from the most stable (lowest $M$ value) to the least stable (highest $M$ value): SDHA/HPRT, R18S, B2M, UBB, ACTB, RPL32, TFRC, GAPDH (Table 3). All genes displayed a relatively high stability over the three time course samplings, with $M$ values $(\mathrm{M}<0.8)$ far below the accepted limit of 1.5 [9]. The two most stably expressed genes of our pool (SDHA and HPRT) allow an optimal normalization of qRT-PCR data, and the addition of a third HKG (R18S) would not significantly increase the statistical reliability of this calculation $\left(\mathrm{V}_{2 / 3}=0.090\right.$, abundantly below the default cut-off value of 0.15 [9]).

The NormFinder algorithm uses a model-based approach for the estimation of modifications among the HKG expressions, also taking into account variation across subgroups and avoiding artificial selection of co-regulated genes [20]. The results of the NormFinder analysis are shown in Table 4 . This ranking appeared to be slightly different from what obtained using geNorm. GAPDH, TFRC and RPL32 still occupy the lowest positions, while SDHA remains the most stable gene. $\mathrm{ACTB}$ gained the second position stepping over HPRT and R18S defined as the least reliable controls.

BestKeeper measures HKG stability by using a pair-wise correlation analysis of all pairs of candidate genes and calculating the geometric mean of the best candidates $[15,19]$. A preliminary analysis, based on the inspection of raw Ct values, estimated the variation of all HKGs to be compatible with an overall stability in gene expression (Table 5), with SD values lower than 1. All genes were retained for the calculation of the BestKeeper index, which similarly exhibited a moderate SD variation (0.58). BestKeeper allows a comparative analysis across HKGs, by estimating correlations in the expression levels between all the possible candidates. Highly correlated control genes are combined into an index. Afterwards, the pair-wise correlation between genes and the correlation between each 


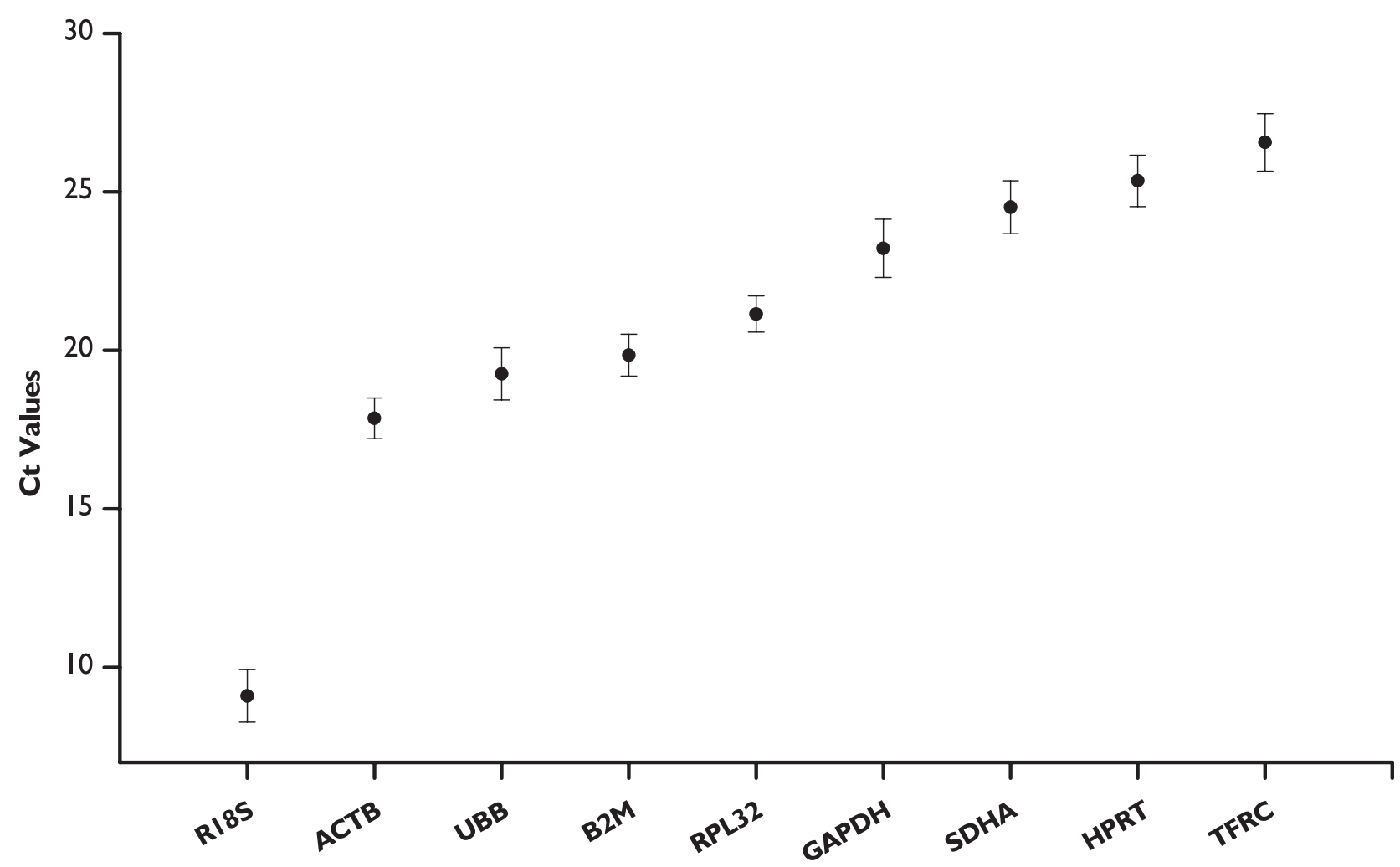

Figure I

Average Ct of candidate HKGs. Expression levels of candidate control genes in the ten endurance horses. Values are given as qRT-PCR cycle threshold numbers (Ct values). Circles represent mean $\mathrm{Ct}$ values, bars indicate the standard deviation.

gene and the index are calculated, describing the consistency between the index and each HKG [15].

The nine control genes tested in our analysis correlated well one with one another and with the BestKeeper index (Table 5). The best correlation between one HKG and the

Table 3: Candidate reference genes ranking according to geNorm.

\begin{tabular}{cccc}
\hline Rank & Gene Symbol & M value & V value \\
\hline $1 / 2$ & SDHA/HPRT & 0.232 & --- \\
3 & $R / 8 S$ & 0.274 & 0.090 \\
4 & B2M & 0.311 & 0.078 \\
5 & UBB & 0.335 & 0.064 \\
6 & ACTB & 0.376 & 0.068 \\
7 & RPL32 & 0.405 & 0.059 \\
8 & TFRC & 0.448 & 0.065 \\
9 & GAPDH & 0.521 & 0.080
\end{tabular}

$M$ value is calculated as average expression stability of control genes during stepwise exclusion of the least stable controls. $V$ value is calculated as the pair-wise variation between two sequential normalization.
BestKeeper index was obtained for SDHA ( $\mathrm{r}=0.983)$, followed by HPRT, R18S and UBB. The statistically significant correlation shown by SDHA with the BestKeeper index appeared to be consistent with the good performance of this gene as assessed by geNorm and NormFinder. It is also remarkable how GAPDH, RPL32 and TFRC, are again classified as the least reliable HKGs, showing the

Table 4: Candidate reference genes ranking according to NormFinder.

\begin{tabular}{ccc}
\hline Rank & Gene Symbol & Stability value \\
\hline 1 & SDHA & 0.056 \\
2 & ACTB & 0.086 \\
3 & $R / 8 S$ & 0.087 \\
4 & HPRT & 0.093 \\
5 & B2M & 0.103 \\
6 & UBB & 0.111 \\
7 & RPL32 & 0.124 \\
8 & TFRC & 0.151 \\
9 & GAPDH & 0.174 \\
\hline
\end{tabular}


worst correlations with the determined BestKeeper index (Table 5).

\section{Discussion}

A number of authors have studied gene expression profiles in exercise induced stress using forefront technologies, like gene chips and qRT-PCR. This study is the first solid contribution in assessing which reference genes have to be used to validate and normalize qRT-PCR outcomes.

Several methods have been proposed to allow accurate normalization of gene expression using qRT-PCR $[9,19$ 22] but at present there is no consensus on which algorithm should be used to measure reference gene stability. A comparison of different methods of reference gene selection allows a better identification of the most reliable controls and reduces the risk of artificial selection of coregulated transcripts [16].

We compared three different statistical approaches (geNorm, NormFinder and BestKeeper) to evaluate nine potential HKGs, in order to select the best reference gene to be used in studying exercise-induced stress in horses.

The uniformity in gene ranking between the three software packages was generally high: SDHA is the most stable HKG according to all the three methods. HPRT similarly displays a constant significant stability. R18S always ranks third, $B 2 M$ fourth and $U B B$ fifth, and can be therefore considered plausible HKGs, even if the addition of supplementary reference genes would not significantly enhance the reliability of the normalization according to the geNorm analysis (V value, Table 3 ).

Regarding $A C T B$, it is difficult to formulate a final judgement because, as already reported in a previous study [15], its classification is not consistent between the three software packages $\left(6^{\text {th }}\right.$ in $g e N o r m$ and BestKeeper, $2^{\text {nd }}$ in NormFinder). Nevertheless, this gene shows an overall reduced variability, as attested by the $M$ value calculated by geNorm and by its good correlation with the BestKeeper index $(r=0.890)$.

GAPDH, TFRC and RPL32 were classified as the least stable genes and they are not likely to be useful in this given experimental system. Notably, the expression of GAPDH, that has been used as HKG in a previous exercise induced stress study [5], appears to be the least stable.

In contrast with what reported elsewhere [23-26], R18S appears to be a good potential reference gene. Despite its good performance, the usefulness of this gene as a control is often doubted: some authors [27] tend to consider it unsuitable for normalization because its transcription is carried out by RNA polymerase I and because of its well known over-expression in comparison with mRNAs (as confirmed even in our experiments, Figure 1). Considering that HKGs that have expression levels comparable to the gene of interest are generally preferred [15], its usage should be carefully considered if used for the normalization of genes that exhibit low level of expression.

\section{Conclusion}

Our results indicate SDHA and HPRT as the most stable reference genes with a very good statistical reliability according to all the three software employed. Moreover, the use of only two genes (SDHA and HPRT) appears to be sufficient for a reliable normalization of the genes of interest; this result is of special interest for future high throughput applications of the technique.

\section{Methods}

\section{Blood collection, RNA extraction and CDNA synthesis}

Ten horses were chosen among participants to national endurance races $(90-120 \mathrm{~km})$. Blood samples were taken from the jugular vein and collected at three different time points: before, at the end of the race, and 24 hours after the race. Immediately after collection, peripheral blood mononuclear cells (PBMCs) were isolated by the FicollHypaque method (GE Healthcare, Pollards Wood, United

Table 5: Statistical output from BestKeeper analysis.

\begin{tabular}{|c|c|c|c|c|c|c|c|c|c|}
\hline & $A C T B$ & $B 2 M$ & GAPDH & HPRT & $R / 8 S$ & RPL32 & SDHA & TFRC & $U B B$ \\
\hline $\mathrm{N}$ & 30 & 30 & 30 & 30 & 30 & 30 & 30 & 30 & 30 \\
\hline $\mathrm{G}$ Mean [Ct] & 17.85 & 19.84 & 23.20 & 25.34 & 9.07 & 21.15 & 24.51 & 26.54 & 19.25 \\
\hline A Mean [Ct] & 17.86 & 19.85 & 23.22 & 25.35 & 9.10 & 21.15 & 24.52 & 26.56 & 19.26 \\
\hline $\operatorname{Min}[\mathrm{Ct}]$ & 16.92 & 18.55 & 20.99 & 24.11 & 7.97 & 20.10 & 23.22 & 25.11 & 17.94 \\
\hline $\operatorname{Max}[\mathrm{Ct}]$ & 19.23 & 21.86 & 25.05 & 27.76 & 12.02 & 22.42 & 26.91 & 28.88 & 21.46 \\
\hline$S D[ \pm C t]$ & 0.49 & 0.51 & 0.71 & 0.62 & 0.57 & 0.46 & 0.66 & 0.73 & 0.64 \\
\hline $\mathrm{CV}[\% \mathrm{Ct}]$ & 2.77 & 2.57 & 3.08 & 2.43 & 6.28 & 2.18 & 2.70 & 2.75 & 3.35 \\
\hline coeff. of corr. [r] & 0.890 & 0.921 & 0.708 & $0.963^{*}$ & $0.949 *$ & 0.832 & $0.983^{*}$ & 0.844 & $0.935^{*}$ \\
\hline $\mathrm{P}$-value & 0.001 & 0.001 & 0.001 & 0.001 & 0.001 & 0.001 & 0.001 & 0.001 & 0.001 \\
\hline
\end{tabular}

Genes are listed in alphabetical order. N: number of samples; $\mathrm{G}$ Mean [Ct]: geometric mean of the $\mathrm{Ct}$; $\mathrm{A}$ Mean [Ct]: arithmetic mean of the $\mathrm{Ct}$; Min and $\operatorname{Max}[\mathrm{Ct}]$ : extreme values of the $\mathrm{Ct}$; SD [Ct]: standard deviation of the $\mathrm{Ct} ; \mathrm{CV}[\% \mathrm{Ct}]$ : coefficient of variance expressed as percentage on the $\mathrm{Ct}$ level. * Indicates the best correlation between control genes and the BestKeeper index. 
Kingdom) from $8 \mathrm{ml}$ of blood. Total RNA was extracted from approximately $1 \times 10^{7}$ PBMCs, using the Aurum Total RNA Fatty and Fibrous Tissue kit (Bio-Rad, Herculers CA, USA) according to the manufacturer's instructions. Genomic DNA was eliminated by a DNase treatment supplied with the kit. Extracted RNA was quantified using the Quant-It RNA assay (Invitrogen, Dorset, United Kingdom) in a VersaFluor fluorometer (Bio-Rad) and checked for integrity in a denaturing agarose gel electrophoresis with ethidium bromide staining. Successful removal of DNA contaminants was tested by absence of PCR amplification in the MC1R gene (GenBank accession number X98012, primers from [28]). $1.5 \mu \mathrm{g}$ of total RNA were retro-transcribed using random hexamers and Superscript III Reverse Transcriptase (Invitrogen) according to the manufacturer's specifications. A PCR with ACTB primers (Table 2) was performed on each cDNA to check for successful retro-transcription.

\section{Reference genes selection and primer design}

Nine widely used reference genes were evaluated: $\beta$-actin (ACTB), glyceraldehyde-3P-dehydrogenase (GAPDH), hypoxanthine ribosyltransferase (HPRT1), $\quad \beta$-2microglobin $(B 2 M)$, succinate dehydrogenase complex subunit A (SDHA), transferrin receptor (TFRC), ubiquitin B (UBB), ribosomal protein L32 (RPL32) and 18S ribosomal rRNA (R18S). In order to minimize the possibility of co-regulation, genes were selected from different functional classes.

Primers were designed based on available sequences using the Primer3 software.

Mfold [29] was used to check the chosen sequences to avoid designing primers in the region of template secondary structure; amplicon lengths were optimized to 68/138 bp to ensure optimal polymerization efficiency. Specificity of amplification was confirmed by sequencing.

For each primer pair, a preliminary real-time assay was performed to evaluate the amplification of non-specific products or primer dimer artefacts (Additional files 1, 2, $3,4,5,6,7,8,9)$. Efficiency of RT-PCR (E), slope values, and correlation coefficients $\left(\mathrm{R}^{2}\right)$ were determined (Table 2) using serial 1:4 dilutions of a template cDNA (pooled from the ten individuals studied, previously diluted 1:5).

PCR products were subsequently resolved on $2 \%$ agarose gel to check for size specificity of the amplicon.

\section{Real-time quantitative PCR}

Five microliters of cDNA template (previously diluted 1:10) were added to the master mix FastStart SYBR Green Master (Roche Applied Science, Penzberg, Germany) with the ROX fluorochrome internal check. PCR reactions, in a volume of $25 \mu \mathrm{l}$ were performed on a MX3000P machine (Stratagene, La Jolla CA, USA). PCR conditions were the same for all primer pairs: initial denaturation at $95^{\circ} \mathrm{C}$ for $10^{\prime}$ followed by 40 cycles of denaturation at $95^{\circ} \mathrm{C}$ for $30^{\prime \prime}$, annealing at $58^{\circ} \mathrm{C}$ for $30^{\prime \prime}$ and extension at $72^{\circ} \mathrm{C}$ for $30^{\prime \prime}$. Fluorescence data were collected at the end of the extension step. Following cycling, the melting curve was determined in the range $58^{\circ}-95^{\circ} \mathrm{C}$, with a temperature slope of $0.01^{\circ} \mathrm{C} / \mathrm{sec}$. Each reaction was run in triplicate with appropriate negative controls.

Baseline and threshold values were automatically determined for all plates and genes using the MxPro software ver. 3.20 (Stratagene). In order to ensure comparability between data obtained from different experimental plates, threshold values for each gene were manually set to the arithmetic mean between the thresholds as automatically determined following each run. Corrected $\mathrm{Ct}$ values were transformed to quantities based on the comparative $\mathrm{Ct}$ method. Following appropriate formatting, values were imported into geNorm (version 3.4), NormFinder (version 0.953) and BestKeeper (version 1) VBA applets.

\section{Authors' contributions}

$\mathrm{KC}, \mathrm{MF}$, SC performed all experiments and data analysis, and drafted the manuscript. GS supervised the study design and the data analysis. MS conceived the project, AVS supervised and coordinated the project and participated in writing the manuscript. All authors read and approved the final manuscript.

\section{Additional material}

\section{Additional file 1}

Melting curve ACTB gene. Melting curve analyses image (jpg format) collected using using the MxPro software ver. 3.20 (Stratagene) during calibration experiments of the selected primer pair for the ACTB gene. Click here for file

[http://www.biomedcentral.com/content/supplementary/14712199-9-49-S1.jpeg]

\section{Additional file 2}

Melting curve B2M gene. Melting curve analyses image (jpg format) collected using using the MxPro software ver. 3.20 (Stratagene) during calibration experiments of the selected primer pair for the $\mathrm{B} 2 \mathrm{M}$ gene. Click here for file

[http://www.biomedcentral.com/content/supplementary/14712199-9-49-S2.jpeg]

\section{Additional file 3}

Melting curve GAPDH gene. Melting curve analyses image (jpg format) collected using using the MxPro software ver. 3.20 (Stratagene) during calibration experiments of the selected primer pair for the GAPDH gene. Click here for file

[http://www.biomedcentral.com/content/supplementary/14712199-9-49-S3.jpeg] 


\section{Additional file 4}

Melting curve HPRT gene. Melting curve analyses image (jpg format) collected using using the MxPro software ver. 3.20 (Stratagene) during calibration experiments of the selected primer pair for the HPRT gene. Click here for file

[http://www.biomedcentral.com/content/supplementary/14712199-9-49-S4.jpeg]

\section{Additional file 5}

Melting curve R18S gene. Melting curve analyses image (jpg format) collected using using the MxPro software ver. 3.20 (Stratagene) during calibration experiments of the selected primer pair for the R18S gene. Click here for file

[http://www.biomedcentral.com/content/supplementary/14712199-9-49-S5.jpeg]

\section{Additional file 6}

Melting curve RPL32 gene. Melting curve analyses image (jpg format) collected using using the MxPro software ver. 3.20 (Stratagene) during calibration experiments of the selected primer pair for the RPL32 gene. Click here for file

[http://www.biomedcentral.com/content/supplementary/14712199-9-49-S6.jpeg]

\section{Additional file 7}

Melting curve SDHA gene. Melting curve analyses image (jpg format) collected using using the MxPro software ver. 3.20 (Stratagene) during calibration experiments of the selected primer pair for the SDHA gene. Click here for file

[http://www.biomedcentral.com/content/supplementary/14712199-9-49-S7.jpeg]

\section{Additional file 8}

Melting curve TFRC gene. Melting curve analyses image (jpg format) collected using using the MxPro software ver. 3.20 (Stratagene) during calibration experiments of the selected primer pair for the TFRC gene. Click here for file [http://www.biomedcentral.com/content/supplementary/14712199-9-49-S8.jpeg]

\section{Additional file 9}

Melting curve UBB gene. Melting curve analyses image (jpg format) collected using using the MxPro software ver. 3.20 (Stratagene) during calibration experiments of the selected primer pair for the UBB gene. Click here for file [http://www.biomedcentral.com/content/supplementary/14712199-9-49-S9.jpeg]

\section{Acknowledgements}

Work sponsored by MIUR - PRIN 2006 (coordinator Prof. Maurizio Silvestrelli)

The authors thank Mr Gianluca Alunni for his valuable technical support and Dr. Francesco Nardi for his suggestions in writing this manuscript. We are also grateful with the horse breeders: Mr Giuseppe Sereni, Mr. Carlo Formica, Miss Chiara Rosi for allowing collection of samples and Dr. Marcello Conte for his kind help.

\section{References}

I. Hamlin MJ, Shearman JP, Hopkins WG: Changes in physiological parameters in overtrained Standardbred racehorses. Equine Veterinary Journal 2002, 34:383-388.

2. Angeli A, Minetto $M$, Dovio A, Paccotti P: The overtraining syndrome in athletes: A stress-related disorder. Journal of Endocrinological Investigation 2004, 27:603-6I2.

3. Cappelli K, Verini-Supplizi A, Capomaccio S, Silvestrelli M: Analysis of peripheral blood mononuclear cells gene expression in endurance horses by cDNA-AFLP technique. Research in Veterinary Science 2007, 82:335-343.

4. Barrey E, Mucher E, Robert C, Amiot F, Gidrol X: Gene expression profiling in blood cells of endurance horses completing competition or disqualified due to metabolic disorder. Equine Veterinary Journal Supply 2006, 36:43-49.

5. Buttner P, Mosig S, Lechtermann A, Funke H, Mooren FC: Exercise affects the gene expression profiles of human white blood cells. Journal of Applied Physiology 2007, I 02:26-36.

6. Zieker D, Fehrenbach E, Dietzsch J, Fliegner J, Waidmann M, Nieselt K, Gebicke-Haerter P, Spanagel R, Simon P, Niess AM, Northoff H: cDNA microarray analysis reveals novel candidate genes expressed in human peripheral blood following exhaustive exercise. Physiological Genomics 2005, 23:287-294.

7. Connolly PH, Caiozzo VJ, Zaldivar F, Nemet D, Larson J, Hung SP, Heck JD, Hatfield GW, Cooper DM: Effects of exercise on gene expression in human peripheral blood mononuclear cells. Journal of Applied Physiology 2004, 97:|46|-|469.

8. Huggett J, Dheda K, Bustin S, Zumla A: Real-time RT-PCR normalisation; strategies and considerations. Genes and Immunity 2005, 6:279-284.

9. Vandesompele J, De Preter K, Pattyn F, Poppe B, Van Roy N, De Paepe A, Speleman F: Accurate normalization of real-time quantitative RT-PCR data by geometric averaging of multiple internal control genes. Genome Biology 2002, 3:research0034-research0034.

10. Selvey S, Thompson EW, Matthaei K, Lea RA, Irving MG, Griffiths LR: Beta-Actin - An unsuitable internal control for RT-PCR. Molecular and Cellular Probes 200 I, I 5:307-3II.

II. Barber RD, Harmer DW, Coleman RA, Clark BJ: GAPDH as a housekeeping gene: Analysis of GAPDH mRNA expression in a panel of $\mathbf{7 2}$ human tissues. Physiological Genomics 2005, 2 I:389-395

12. Bas A, Forsberg G, Hammarstrom S, Hammarstrom ML: Utility of the housekeeping genes I8S rRNA, beta-actin and glyceraldehyde-3-phosphate-dehydrogenase for normalization in real-time quantitative reverse transcriptase-polymerase chain reaction analysis of gene expression in human $\mathbf{T}$ lymphocytes. Scandinavian Journal of Immunology 2004, 59:566-573.

13. Dheda K, Huggett JF, Bustin SA, Johnson MA, Rook G, Zumla A: Validation of housekeeping genes for normalizing RNA expression in real-time PCR. Biotechniques 2004, 37: I I2-II9.

14. Banda M, Bommineni A, Thomas RA, Luckinbill LS, Tucker JD: Evaluation and validation of housekeeping genes in response to ionizing radiation and chemical exposure for normalizing RNA expression in real-time PCR. Mutation Research 2007.

15. Spinsanti G, Panti C, Lazzeri E, Marsili L, Casini S, Frati F, Fossi CM: Selection of reference genes for quantitative RT-PCR studies in striped dolphin (Stenella coeruleoalba) skin biopsies. Bmc Molecular Biology 2006, 7:.

16. Ayers D, Clements D, Salway F, Day P: Expression stability of commonly used reference genes in canine articular connective tissues. BMC Veterinary Research 2007, 3:.

17. Maccoux LJ, Clements DN, Salway F, Day PJR: Identification of new reference genes for the normalisation of canine osteoarthritic joint tissue transcripts from microarray data. $\mathrm{Bmc}$ Molecular Biology 2007, 8:

18. Bogaert L, Van Poucke M, De Baere C, Peelman L, Gasthuys F, Martens A: Selection of a set of reliable reference genes for quantitative real-time $P C R$ in normal equine skin and in equine sarcoids. Bmc Biotechnology 2006, 6:

19. Pfaffl MW, Tichopad A, Prgomet C, Neuvians TP: Determination of stable housekeeping genes, differentially regulated target genes and sample integrity: BestKeeper - Excel-based tool using pair-wise correlations. Biotechnology Letters 2004, 26:509-5 I5. 
20. Andersen CL, Jensen JL, Orntoft TF: Normalization of real-time quantitative reverse transcription-PCR data: $A$ model-based variance estimation approach to identify genes suited for normalization, applied to bladder and colon cancer data sets. Cancer Research 2004, 64:5245-5250.

21. Akilesh S, Shaffer DJ, Roopenian D: Customized molecular phenotyping by quantitative gene expression and pattern recognition analysis. Genome Research 2003, 13:1719-1727.

22. Haller F, Kulle B, Schwager S, Gunawan B, Heydebreck AV, Itmann H, zesi $L$ : Equivalence test in quantitative reverse transcription polymerase chain reaction: Confirmation of reference genes suitable for normalization. Analytical Biochemistry 2004, 335: I-9.

23. Blanquicett $C$, Johnson MR, Heslin M, Diasio RB: Housekeeping gene variability in normal and carcinomatous colorectal and liver tissues: Applications in pharmacogenomic gene expression studies. Analytical Biochemistry 2002, 303:209-2/4.

24. Thellin O, Zorzi W, Lakaye B, De Borman B, Coumans B, Hennen G, Grisar T, Igout A, Heinen E: Housekeeping genes as internal standards: Use and limits. Journal of Biotechnology 1999, 75:29I-295.

25. Schmittgen TD, Zakrajsek BA: Effect of experimental treatment on housekeeping gene expression: Validation by real-time, quantitative RT-PCR. Journal of Biochemical and Biophysical Methods 2000, 46:69-8I.

26. Bond BC, Virley DJ, Cairns NJ, Hunter AJ, Moore GBT, Moss SJ, Mudge AW, Walsh FS, Jazin E, Preece P: The quantification of gene expression in an animal model of brain ischaemia using TaqMan real-time RT-PCR. Molecular Brain Research 2002, 106:101-116.

27. Radonic A, Thulke S, Mackay IM, Landt O, Siegert W, Nitsche A: Guideline to reference gene selection for quantitative realtime PCR. Biochemical and Biophysical Research Communications 2004, 3 I 3:856-862.

28. Rieder S, Taourit S, Mariat D, Langlois B, Guerin G: Mutations in the agouti (ASIP), the extension (MCIR), and the brown (TYRPI) loci and their association to coat color phenotypes in horses (Equus caballus). Mammalian Genome 200I, 12:450-455.

29. Zuker M: Mfold web server for nucleic acid folding and hybridization prediction. Nucleic Acids Research 2003, 31:3406-34 I5.

Publish with Bio Med Central and every scientist can read your work free of charge

"BioMed Central will be the most significant development for disseminating the results of biomedical research in our lifetime. "

Sir Paul Nurse, Cancer Research UK

Your research papers will be:

- available free of charge to the entire biomedical community

- peer reviewed and published immediately upon acceptance

- cited in PubMed and archived on PubMed Central

- yours - you keep the copyright
BioMedcentral 